\title{
Assessment of the Efficiency \\ of Transformers Technical Condition Monitoring Based on Dissolved Gas Analysis
}

\author{
Ammar A. Yahya and Vladimir M. Levin* \\ Novosibirsk State Technical University \\ Novosibirsk, Russian Federation
}

Received 18.02.2020, received in revised form 20.02.2020, accepted 25.02.2020

\begin{abstract}
The method of analysis of gases dissolved in oil is one of the most informative methods for early detection of defects in power oil-filled transformers. This determined its wide application in the practice of off-line and on-line monitoring of transformer equipment. In the article, based on the ratio of weight coefficients used in the calculation of the technical condition index of functional units and equipment units, a method for determining the reliability of oil-filled transformer equipment is proposed. An indicator that characterizes the quantitative measure of the diagnostic value of a single method (group of methods) for monitoring parameters of a power oil-filled transformer in the calculations of the technical condition index is obtained. The efficiency of monitoring the state of a power oil-filled transformer was evaluated based on the results of the analysis of gases dissolved in oil. A quantitative example of calculating the economic effect of increasing the operational reliability of a group of $110 \mathrm{kV}$ power transformers in one of the areas of the distribution network using a remote diagnostic monitoring system based on the analysis of gases dissolved in oil is given.
\end{abstract}

Keywords: monitoring, transformer, dissolved gas analysis, technical condition index, economic efficiency, operational reliability.

Citation: Yahya A.A., Levin V.M. Assessment of the efficiency of transformers technical condition monitoring based on dissolved gas analysis, J. Sib. Fed. Univ. Eng. \& Technol., 2020, 13(4), 438-448. DOI: 10.17516/1999-494X-0235

(C) Siberian Federal University. All rights reserved

This work is licensed under a Creative Commons Attribution-Non Commercial 4.0 International License (CC BY-NC 4.0).

* Corresponding author E-mail address: levin@corp.nstu.ru 


\title{
Оценка эффективности мониторинга
}

\section{технического состояния трансформаторов \\ на основе анализа растворенных газов}

\author{
А.А. Яхья, В.М. Левин \\ Новосибирский государственный технический университет \\ Российская Федерация, Новосибирск
}

\begin{abstract}
Аннотация. Метод анализа растворенных в масле газов относится к одному из наиболее информативных методов раннего обнаружения дефектов в силовых маслонаполненных трансформаторах. Это определило его беспрецедентно широкое применение в практике offline- и on-line-мониторинга трансформаторного оборудования. В статье на основе соотношений весовых коэффициентов, используемых в расчетах индекса технического состояния функциональных узлов и единицы оборудования, предложена методика определения показателей безотказности силового маслонаполненного трансформатора. Получен показатель, характеризующий количественную меру диагностической ценности единичного метода (группы методов) мониторинга параметров силового маслонаполненного трансформатора в расчетах индекса технического состояния. Выполнена оценка эффективности мониторинга состояния силового маслонаполненного трансформатора по результатам анализа растворенных в масле газов. Приведен числовой пример расчета экономического эффекта от повышения эксплуатационной надежности группы силовых трансформаторов 110 кВ одного из районов распределительной электрической сети с использованием системы удаленного мониторинга и диагностики на основе анализа растворенных в масле газов.
\end{abstract}

Ключевые слова: мониторинг, трансформатор, анализ растворенных газов, индекс технического состояния, экономическая эффективность, эксплуатационная надежность.

Цитирование: Яхья, А.А. Оценка эффективности мониторинга технического состояния трансформаторов на основе анализа растворенных газов / А.А. Яхья, В.М. Левин // Журн. Сиб. федер. ун-та. Техника и технологии, 2020. 13(4). С. 438-448. DOI: 10.17516/1999-494X-0235

\section{Введение}

Силовой маслонаполненный трансформатор (СМТ) представляет собой сложное, ответственное и весьма дорогостоящее техническое устройство, которое широко применяется в составе оборудования подстанций генерирующих и сетевых объектов электроэнергетической системы. Сложная конструкция СМТ включает ряд функциональных узлов (конструктивных элементов) $)^{1}$, что обеспечивает ему возможность выполнения необходимых функций: преобразование электрического тока в магнитное поле, распределение тока (мощности) нагрузки между обмотками, трансформация (повышение / понижение) напряжения, регулирование уровня напряжения.

Для надежной работы СМТ предназначен комплекс методов диагностирования и оценки технического состояния, позволяющий выявлять неисправности (дефекты) в отдельных функциональных узлах по мере их возникновения [1]. Каждый метод диагностирования име-

Силовые трансформаторы. Справочная книга. Под ред. С.Д. Лизунова, А.К. Лоханина. М.: Энергоатомиздат, 2004, 616 c. [Power transformer. Reference book. Pod red. S.D. Lizunova, A.K. Lohanina. M.: Energoatomizdat, 2004, 616 p. (in Russian)].

$$
-439-
$$


ет свое адресное назначение и ориентирован на обнаружение дефектов в конкретном конструктивном элементе СМТ. Методы диагностирования различаются информативностью, а также условиями и периодичностью применения ${ }^{2}$. Большинство из них применяют off-line, т.е. на отключенном и расшинованном трансформаторе. Это вызывает ряд неудобств, так как зачастую требует ограничения в электроснабжении потребителей. Кроме того, off-lineдиагностирование имеет относительно низкую информативность, поскольку оперирует с откликами на тестовые сигналы, обладающие, как правило, более низкими параметрами по сравнению с рабочими (например, методы измерения потерь холостого хода $\Delta P_{\mathrm{X}}$ или сопротивления короткого замыкания $\Delta Z_{\mathrm{K}}$ ). Особую группу составляют методы раннего обнаружения дефектов в СMT, которые применяют в режиме on-line на работающем нагруженном трансформаторе. К ним относится анализ растворенных в масле газов (АРГ), физико-химический анализ масла (ФХА), инфракрасный контроль (ИК), виброконтроль (ВК), контроль частичных разрядов (ЧР) [2].

Метод АРГ основан на измерении концентраций диагностических газов, растворенных в масле СМТ, и признан наиболее информативным. Он позволяет обеспечить выявление до 70 \% дефектов в элементах активной части (обмотки, магнитопровод, контактные соединения, целлюлозная изоляция), а также в масле [3-6]. Это определило его беспрецедентно широкое применение в практике off-line- и on-line-мониторинга маслонаполненного трансформаторного оборудования 35-110 кВ и выше.

Одним из векторов развития распределительных электрических сетей в направлении повышения эксплуатационной надежности и информационной безопасности основного технологического оборудования, к которому принадлежат СМТ, является распространение систем удаленного мониторинга и диагностики (СУМиД) [7, 8]. Принципы создания СУМиД базируются на интеграции информационных и вычислительных ресурсов объектов распределительной электрической сети с целью сбора, обработки и передачи данных о текущих параметрах состояния СМТ каждой из подстанций для последующей оценки технического состояния и выбора адекватного вида и стоимости эксплуатационных воздействий на объект. Ключевыми функциями СУМиД считаются:

- технологический мониторинг основного технологического оборудования (раннее обнаружение дефектов, оценка остаточного ресурса, прогнозирование вероятности наступления аварийных событий, отказов);

- удаленное управление основным технологическим оборудованием с возможностью удаленного воздействия по изменению его функциональных параметров либо отключению.

Эффективность функционирования СУМиД определятся информативностью применяемых методов мониторинга и диагностирования СМТ. В связи с этим актуальна оценка эффективности каждого из методов, которыми оснащена СУМиД, с расчетом экономического эффекта от повышения эксплуатационной надежности объектов распределительной электрической сети.

2 СТО 34.01-23.1-001-2017 Объем и нормы испытаний электрооборудования. Стандарт организации ПАО «Россети» М.: ПАО «Россети», 2017. [Электронный ресурс]. - Режим доступа: https://files.stroyinf.ru/Data2/1/4293734154. pdf. - Заглавие с экрана [Scope and standards for testing electrical equipment. Standard of organization of PJSC «Rosseti» [Electronic resource] - Access: https://files.stroyinf.ru/Data2/1/4293734154.pdf (in Russia)]. 


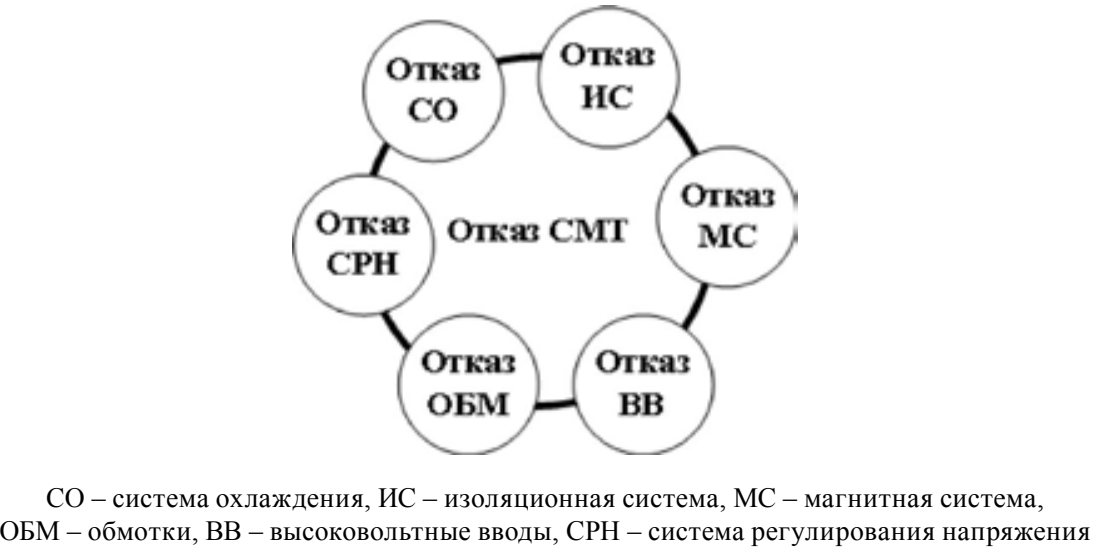

Рис. 1. События, приводящие к отказу СМТ

Fig. 1. Events that lead to POFT failure

\section{Моделирование отказов СМТ с учетом его технического состояния}

Отказ СМТ представляет собой сложное случайное событие, состоящее из ряда независимых и совместных элементарных случайных событий, а именно отказов функциональных узлов по причине развития дефекта (рис. 1). Аварийным дефектом оборудования принято считать дефект, вызванный неумелым его обслуживанием и неправильной эксплуатацией. К появлению аварийного дефекта, при котором возникает отказ функционального узла, могут приводить несколько причин: ошибки при диагностировании, несвоевременное обнаружение признаков неисправности, высокая скорость развития дефекта.

Структурная схема надежности СМТ - последовательное соединение входящих в его состав элементов (функциональных узлов). В указанном случае вероятность отказа СMT ( $\left.Q_{\text {смт }}\right)$ определяют на основе теоремы умножения вероятностей по формуле

$$
Q_{\mathrm{cMT}}=1-\prod_{i=1}^{n}\left(1-Q_{i}\right),
$$

где $n$ и $Q_{i}$ - количество и вероятность отказа $i$-го функционального узла СМТ. Для определения $Q_{i}$ требуется проведение статистических вычислений с привлечением ретроспективных данных о наступлении аварийных событий для каждого функционального узла СМТ, что представляется весьма проблематичным. В то же время доступной и вполне достоверной информацией является значение среднестатистической интенсивности отказа СМТ ( $\left.\bar{\omega}_{0}\right)$ определенного класса номинального напряжения [9], а кроме того, значения весовых коэффициентов функциональных узлов $\left(B_{i}, i=\overline{1, n}\right)$, характеризующих важность каждого из них с точки зрения обеспечения эксплуатационной надежности СМТ [10].

В практике диагностирования СМТ за каждым функциональным узлом закреплена группа контролируемых параметров состояния и методов их диагностирования. Весовой коэффициент $j$-й группы контролируемых параметров $i$-го функционального узла $\left(b_{i j}, j=\overline{1, m} ; i=\overline{1, n}\right)$ характеризует его важность с точки зрения надежности последнего. При этом сумма $b_{i j}$ равна 1 . В табл. 1 приведены группы параметров состояния каждого функци-

$$
-441-
$$


Таблица 1. Параметры состояния функциональных узлов СМТ и их весовые коэффициенты

Table 1. State parameters of POFT functional nodes and their weight coefficients

\begin{tabular}{|c|c|c|c|}
\hline $\begin{array}{c}\text { № } \\
\text { П.П. }\end{array}$ & $\begin{array}{c}\text { Функциональный } \\
\text { узел }\end{array}$ & $\begin{array}{c}\text { Группа контролируемых параметров } \\
\text { (методов диагностирования) }\end{array}$ & $\begin{array}{c}\text { Весовой } \\
\text { коэф-нт, } b_{i j}\end{array}$ \\
\hline 1 & $\begin{array}{l}\text { Изоляционная } \\
\text { система }\end{array}$ & $\begin{array}{l}\text { АРГ (концентрации газов) } \\
\text { ФХА (пробивное напряжение, } \operatorname{tg} \delta_{\text {масла }}, \text { др.) } \\
\text { Состояние целлюлозы }\left(\mathrm{W}_{\text {цел., }} \text { сод. фуран. пр-х) }\right. \\
\text { Отношение концентр. пар характерных газов }\end{array}$ & $\begin{array}{l}0,425 \\
0,425 \\
0,140 \\
0,010\end{array}$ \\
\hline 2 & Магнитная система & $\begin{array}{l}\text { Локальный нагрев бака по результатам ТВК } \\
\text { Потери холостого хода }\left(\Delta P_{\mathrm{x}}\right)\end{array}$ & $\begin{array}{l}0,500 \\
0,500\end{array}$ \\
\hline 3 & Обмотки & $\begin{array}{l}\text { Состояние геометрии обмоток }\left(\Delta Z_{\mathrm{x}}\right) \\
\text { Тенденции изменения } \operatorname{tg} \delta_{\text {цел. }}, R_{60}(\text { по ср. с нач.) } \\
\mathrm{R} \text { постоянному току обмоток } \mathrm{BH}, \mathrm{HH}\end{array}$ & $\begin{array}{l}0,010 \\
0,500 \\
0,490\end{array}$ \\
\hline 4 & $\begin{array}{l}\text { Высоковольтные } \\
\text { вводы }\end{array}$ & $\begin{array}{l}\text { Состояние изоляции }(\operatorname{tg} \delta, R, C) \\
\text { АРГ (концентрации газов) } \\
\text { ФХА (пробивное напряжение, } \operatorname{tg} \delta_{\text {масла }}, \text { др.) } \\
\text { Целостность конструкции (давление, } \theta^{\circ} \mathrm{C}, \text { др.) }\end{array}$ & $\begin{array}{l}0,250 \\
0,250 \\
0,250 \\
0,250\end{array}$ \\
\hline 5 & $\begin{array}{l}\text { Система рег-ния } \\
\text { напряжения (РПН) }\end{array}$ & $\begin{array}{l}\text { Состояние масла: пробивное напряжение, } \mathrm{W}_{\text {масла }} \\
\text { Состояние механизмов привода и контактора }\end{array}$ & $\begin{array}{l}0,334 \\
0,666\end{array}$ \\
\hline 6 & $\begin{array}{l}\text { Система } \\
\text { охлаждения }\end{array}$ & $\begin{array}{l}\text { Состояние бака и навесного оборудования } \\
\text { Состояние защитного оборудования }\end{array}$ & $\begin{array}{l}0,500 \\
0,500\end{array}$ \\
\hline
\end{tabular}

онального узла СМТ, методы их диагностирования и значения соответствующих весовых коэффициентов.

Вероятность сложного события, образующего полную группу простых несовместных случайных событий, может быть рассчитана на основе теоремы сложения вероятностей по формуле

$$
Q_{\text {смт }}=\sum_{i=1}^{n} Q_{i}=\sum_{i=1}^{n}\left(1-\mathrm{e}^{-\overline{\omega_{0}} \cdot B_{i} \cdot t}\right) .
$$

Модель отказа (2) не учитывает индивидуальных характеристик старения функциональных узлов СМТ и справедлива лишь для интервала $t$ их нормальной эксплуатации. При необходимости дифференциации СМТ в группе по сроку службы (остаточному ресурсу) целесообразно применение более сложной модели отказа, например полученной в [11]. В табл. 2 приведены значения весовых коэффициентов и вероятностей отказа функциональных узлов, рассчитанных по модели (2) для СМТ 110 кВ при значении $\bar{\omega}_{0}=0,015$ (год ${ }^{-1}$ ).

Как следует из результатов табл. 2, наибольшая вероятность отказа принадлежит изоляционной системе СМТ, что полностью согласуется с информацией из открытых источников. Приведенные результаты расчета вероятностей отказа функциональных узлов СМТ получены для условия своевременного выявления и устранения дефектов вследствие мониторинга (диагностирования) и приложения адекватных ремонтных воздействий. Очевидно, что отсутствие в составе программно-аппаратного комплекса СУМиД метода контроля одного или нескольких параметров состояния функциональных узлов СМТ изменит соотношение соответствующих вероятностей отказа в сторону их увеличения. 
Таблица 2. Значения $B_{i}$ и $Q_{i}$ функциональных узлов СМТ

Table 2. The values of $B_{i}$ and $Q_{i}$ functional units of the POFT

\begin{tabular}{|c|l|c|c|}
\hline № п.п. & \multicolumn{1}{|c|}{ Функциональный узел } & $B_{i}$, о.е. & $Q_{i}$, о.е. \\
\hline 1 & Изоляционная система & 0,32 & 0,0048 \\
\hline 2 & Магнитная система & 0,18 & 0,0027 \\
\hline 3 & Обмотки & 0,18 & 0,0027 \\
\hline 4 & Высоковольтные вводы & 0,13 & 0,0019 \\
\hline 5 & Система регулирования напряжения & 0,10 & 0,0015 \\
\hline 6 & Система охлаждения & 0,09 & 0,0013 \\
\hline ИТОГО & & 1,00 & 0,0149 \\
\hline
\end{tabular}

\section{Критерий оченки эффективности СУМиД с применением АРГ СМТ}

Оценим эффективность мониторинга (диагностирования) СМТ на основе АРГ. Для этого рассмотрим два противоположных случая. В первом случае программно-аппаратный комплекс СУМиД объектов распределительной электрической сети имеет в своем составе методы мониторинга (диагностирования) параметров СМТ согласно табл. 1. Во втором случае в состав методов не включен АРГ.

Мониторинг СМТ 110 кВ на основе АРГ позволяет выявить дефекты сразу нескольких функциональных узлов трансформатора на ранней стадии развития с последующим их устранением и таким образом понизить общую вероятность отказа СМТ за счет снижения вероятностей отказа этих функциональных узлов. Из данных табл. 1 следует, что к функциональным узлам, для которых АРГ формирует группу контролируемых параметров, относятся ИС и ВВ.

В расчетах индекса технического состояния (ИТС) функциональных узлов и единицы оборудования применяется система весовых коэффициентов. Она позволяет ранжировать контролируемый параметр либо функциональный узел в зависимости от его относительной важности среди подобных для обеспечения работоспособности оборудования [10].

Введем в рассмотрение индекс $D$, который будет характеризовать диагностическую ценность мониторинга параметров состояния функциональных узлов с применением комплекса методов. Количественную оценку $D, \%$ можно получить с помощью соотношения весовых коэффициентов $B_{i}$ и $b_{i j}$ по выражению

$$
D=100 \cdot \sum_{i=1}^{n}\left(\frac{B_{i}}{\sum_{j=1}^{m} b_{i j}}\right), \% .
$$

При условии, что в расчете ИТС трансформатора принимают участие все группы контролируемых параметров состояния функциональных узлов, значение $D$ по формуле (3) получается равным $100 \%$. Если же в расчете ИТС отсутствует информация по какой-либо группе параметров / методу мониторинга (диагностирования), значения соответствующих $b_{i j}=0$, a

$$
-443-
$$


$D>100 \%$. Тогда количественную оценку конкретного метода мониторинга (диагностирования) в расчете ИТС СМТ можно получить следующим образом:

$$
\Delta D=(D-100), \% .
$$

Для метода АРГ при подстановке соответствующих значений $b_{i j}$ из табл. 1 и 2 в формулу (3) получим $\Delta D=27,32 \%$. Таким образом, вероятность отказа функциональных узлов СМТ без применения в СУМиД метода АРГ увеличится и составит $Q_{\text {смт }}^{\prime}=0,0149 \cdot 1,2732=0,01897$. В табл. 3 приведены значения $Q_{i}^{\prime}$ вероятностей отказа функциональных узлов СМТ 110 кВ без

Таблица 3. Значения вероятностей $Q_{i}^{\prime}$ без применения метода АРГ

Table 3. Of probabilities $Q_{i}^{\prime}$ without the use of the DGA method

\begin{tabular}{|l|c|c|c|c|c|c|}
\hline Функциональный узел & ИС & МС & ОБМ & ВВ & СРН & СО \\
\hline$Q_{i}^{\prime}$ & 0,00602 & 0,00338 & 0,00338 & 0,00244 & 0,00188 & 0,00169 \\
\hline
\end{tabular}

применения мониторинга (диагностирования) по методу АРГ, полученные с помощью выражения (3). Эффект от применения мониторинга (диагностирования) СМТ по методу АРГ в виде понижения результирующей вероятности отказа составит:

$$
\Delta Q=Q_{\mathrm{cMT}}-Q_{\mathrm{cMT}}^{\prime}=Q_{\mathrm{cMT}} \cdot \frac{\Delta D}{100}=0,0188-0,0149=0,0039 .
$$

\section{Результаты расчетов. Анализ и обсуждение}

Произведем расчет экономии эксплуатационных затрат группы СМТ 110 кВ на интервале ремонтного цикла от изменения уровня надежности, вызванного применением мониторинга (диагностирования) на основе АРГ.

Ремонтным циклом ( $\triangle T$ рц) электрооборудования принято называть интервал эксплуатации между двумя капитальными ремонтами (КР). Согласно справочным данным, ремонтный цикл СМТ 110 кВ составляет 8 лет, интервал между текущими ремонтами (ТР) - 4 года, между техническими обслуживаниями (ТО) - 1 год. Таким образом, структура ремонтного цикла будет следующей:

$$
\Delta T_{\mathrm{pц}}=8 \text { лет }=7 \mathrm{TO}+1 \mathrm{TP} .
$$

Стоимость единичного СМТ типа ТДН-25000/110, по данным завода-изготовителя, составляет 25 млн руб. В табл. 4 приведены значения стоимостей эксплуатационных воздействий на СМТ в \% от стоимости новой единицы оборудования и в рублях.

C учетом данных табл. 4 суммарные затраты на полный ремонтный цикл единичного СМТ 110 кВ без применения мониторинга (диагностирования) на основе метода АРГ таковы:

$$
3_{\mathrm{pu}}=7 \cdot 0,15+1,5=2,55 \text { млн руб. }
$$


Таблица 4. Стоимость эксплуатационного воздействия на СМТ

Table 4. Cost of operational impact on POFT

\begin{tabular}{|c|c|c|}
\hline \multicolumn{3}{|c|}{ Стоимость эксплуатационного воздействия, \% (млн руб.) } \\
\hline КР & TP & TО \\
\hline $10 \%(2,5)$ & $6 \%(1,5)$ & $0,6 \%(0,15)$ \\
\hline
\end{tabular}

Экономия эксплуатационных затрат на ремонтном цикле единичного СМТ 110 кВ от применения мониторинга (диагностирования) на основе АРГ составит:

$$
\Delta 3_{\mathrm{pu}}=3_{\text {рu }} \cdot \frac{\Delta D}{100}=2,55 \cdot \frac{27,32}{100}=0,6967 \text { млн руб. }
$$

На подстанциях рассматриваемого сетевого района эксплуатируются 26 СМТ с номинальным напряжением 110 кВ. С учетом этого суммарная экономия затрат на техническое обслуживание и ремонт всего парка трансформаторов данного класса напряжения будет следующей:

$$
3_{\text {сум. }}=26 \cdot 0,6967=8,1142 \text { млн руб. }
$$

Применение в СУМиД информации по методу АРГ повлечет дополнительные затраты, вызванные приобретением специализированной диагностической техники, ее содержанием и использованием по назначению. В случае off-line-мониторинга (диагностирования) СМТ АРГ выполняют в лабораторных условиях на базе стационарного хроматографа. Рыночная стоимость хроматографа марки «Хроматэк-Кристалл 5000» составляет 2,0 млн руб. Стоимость единичного хроматографического анализа масла СМТ принята из опыта эксплуатации равной 0,0018 млн руб.

Для группы из 26 единиц СМТ 110 кВ на интервале $\Delta T_{\text {рц }}=8$ лет с учетом периодичности АРГ 2 раза в год рассчитаем:

1) суммарную стоимость АРГ:

$$
\mathrm{C}_{\text {АРГ }}=26 \cdot 2 \cdot 8 \cdot 0,0018=0,7488 \text { млн руб.; }
$$

2) суммарные эксплуатационные затраты на off-line-мониторинг (диагностирование) группы СМТ 110 кВ распределительных подстанций по методу АРГ:

$$
3_{\mathrm{AP \Gamma}}=\mathrm{K}_{\mathrm{xp}} \cdot \mathrm{E}+\mathrm{U}_{\Delta T_{\mathrm{p \mu}}}+\mathrm{C}_{\mathrm{AP \Gamma}}=2,0 \cdot 0,15+2,0 \cdot 0,05 \cdot 8+0,7488=1,8488 \text { млн руб., }
$$

где $\mathrm{K}_{\mathrm{xp}}, \mathrm{E}$ - стоимость хроматографа и коэффициент дисконтирования (о.е.); $\mathrm{h}_{\Delta T_{\mathrm{pц}}}=\mathrm{K}_{\mathrm{xp}} \cdot \alpha \cdot \Delta T_{\mathrm{pц}}-$ суммарные издержки на содержание хроматографа на интервале ремонтного цикла (руб.); $\alpha-$ норматив суммарных отчислений (о.е.);

3) экономию затрат от повышения эксплуатационной надежности группы однотипных СМТ подстанций 110 кВ района распределительной сети на интервале $\Delta T_{\text {цц: }}$

$$
Э=\Delta 3_{\text {рц }}-3_{\text {АРГ }}=18,1142-1,8488=16,2654 \text { млн руб. }
$$

Расчет выполнен с некоторыми допущениями, так как не учитывает отдельных ограничивающих факторов. К ним следует отнести:

$$
-445-
$$


1) возможность ошибок при диагностировании по методу АРГ, которые могут быть вызваны качеством отбора проб масла из ВВ и бака СМТ, а также интерпретацией результатов хроматографии;

2) разброс во времени для оценок технического состояния по результатам АРГ каждого единичного СМТ контрольной группы;

3) неодновременность вывода из работы трансформаторов контрольной группы для выполнения плановых операций по техническому обслуживанию и ремонту на интервале ремонтного цикла;

4) колебания ценовых показателей на рынке диагностического и силового оборудования, а также услуг энергосервиса.

Снятие ограничивающих допущений может повлиять на изменение количественных значений рассчитанных показателей в сторону их понижения, однако это не способно изменить качественного соотношения полученных результатов.

Таким образом, несмотря на возможное снижение абсолютного значения экономического эффекта от применения метода АРГ в составе программно-аппаратного комплекса СУМиД подстанций распределительной электрической сети, его относительная эффективность (диагностическая ценность) для повышения эксплуатационной надежности трансформаторного оборудования за счет оперативного выявления и своевременного устранения дефектов принципиально не изменится.

\section{Заключение}

Метод анализа растворенных в масле газов нашел широкое применение в диагностике и оценке технического состояния силового маслонаполненного оборудования благодаря возможности обнаружения дефектов на ранних стадиях развития в рабочих режимах без снятия напряжения. Это определило его достаточно высокий рейтинг, экспертно установленный с помощью весовых коэффициентов для групп контролируемых параметров в расчетах ИТС функциональных узлов и СМТ в целом.

Применение структурных схем надежности к СМТ как к сложной системе позволяет получить модели отказа элементов (функциональных узлов) с учетом фактического технического состояния каждого из них на основе актуальной диагностической информации. Возможности системы весовых коэффициентов, используемых в расчетах ИТС, не ограничиваются ранжированием функциональных узлов СМТ по важности для обеспечения его эксплуатационной надежности, но также позволяют установить количественную меру диагностической ценности метода (группы методов) мониторинга и диагностирования для оценки их эффективности в СУМиД подстанций распределительной электрической сети. Использование индекса диагностической ценности имеет практическое применение при выборе и обосновании минимально необходимого состава программно-аппаратного комплекса СУМиД.

Проведенный расчет экономического эффекта демонстрирует снижение суммарных эксплуатационных затрат группы СМТ 110 кВ района электрической сети на интервале ремонтного цикла от повышения надежности за счет применения СУМиД на основе АРГ и подтверждает начальные предположения авторов. Более строгий учет ограничивающих факторов позволит уточнить расчет экономического эффекта и может уменьшить его количественное значение.

$$
-446-
$$


Однако это принципиально не повлияет на качественное соотношение полученных результатов.

\section{Список литературы / References}

[1] Алексеев Б.А. Контроль состояния (диагностика) крупных силовых трансформаторов. М.: Изд-во НЦ ЭНАС, 2001. 216 с. [Alekseev В.A. State control (diagnostics) of large power transformers. Moscow: Publishing house of NC ENAS. 2001. 216 p. (in Russian)].

[2] Arvind Dh., Khushdeep S., Deepak K. Condition Monitoring of Power Transformer, A Review IEEE, 2008.

[3] De Faria H.Jr., Costa J.G.S., Olivas, J.L.M. A review of monitoring methods for predictive maintenance of electric power transformers based on dissolved gas analysis, Renew. Sustain. Energy Rev., 2015, 46, 201-209.

[4] Bakar N.A., Abu-Siada A.A. New Method to Detect Dissolved Gases in Transformer Oil using NIR-IR Spectroscopy, IEEE Trans. Dielectr. Electr. Insul., 2017, 24, 409-419.

[5] De Faria H.Jr., Costa J.G. S., Olivas J.L.M. A review of monitoring methods for predictive maintenance of electric power transformers based on dissolved gas analysis, Renewable and Sustainable Energy Reviews, 2015, 46, 201-209.

[6] Salam C.A.M., Rahman Q., Wen F., S. Ang, and W. Voon, Causes of transformer failures and diagnostic methods, A review, Renewable and Sustainable Energy Reviews, 2018, 82, 1442-1456.

[7] Смекалов В.В., Волошин А.А., Гусарова А.А. Создание программно-технического комплекса для принятия решения о воздействии на электросетевое оборудование с учетом его технического состояния и индекса важности на основе современных методов диагностики и обработки данных. [Электронный ресурс] - Режим достуnа: https://cis-ees.ru/RUM/eMagazine/ Articles/Details/3109. - Заглавие с экрана [Smekalov V.V., Voloshin A.A., Gusarova A.A. Creating a software and hardware complex for making decisions about the impact on power grid equipment, taking into account its technical condition and importance index, based on modern methods of diagnostics and data processing [Electronic resource] - Access: https://cis-ees.ru/RUM/eMagazine/ Articles/Details/3109 (in Russian)].

[8] Захаров О.А. Цифровизация электросетевого комплекса: пути решения или система прогностики и мониторинга, Руководящие материаль по проектированию и эксплуатации электрических сетей, 2019, 3 (587) [Электронный ресурс] - Режим доступа: https://prana-system.com/ novosti/novosti/cifrovizaciya-elektrosetevogo-kompleksa-puti-resheniya-ili-sistema-prognostikii-monitoringa. - Заглавие с экрана [Zakharov O.A. Digitalization of the electric grid complex: solutions or a system of forecasting and monitoring, Guidelines for the design and operation of electric networks [Electronic resource] - Access: https:/prana-system.com/novosti/novosti/cifrovizaciyaelektrosetevogo-kompleksa-puti-resheniya-ili-sistema-prognostiki-i-monitoringa (in Russian)].

[9] Ящура А.И. Система технического обслуживания и ремонта энергетического оборудования. М.: Изд-во НЦ ЭНАС, 2006. 504 с. [Yashchura A.I. The system of technical maintenance and repair of power equipment. Moscow: Publishing house of NC ENAS. 2006. 504 p. (in Russian)].

[10] Байдюк А.М., Комарова Г.В. Оценка технического состояния и надежности электрических машин, Известия СПбГЭТУ ЛЭТИ, 2019, 3, 78-84 [Bajdyuk A.M., Komarova G.V. Assessment

$$
-447-
$$


of the technical condition and reliability of electric machines, Izvestiya SPbGETU LETI, 3, 78-84 (in Russian)].

[11] Левин В.М. Моделирование потока отказов силовых трансформаторов в условиях эксплуатации, Современные энергетические системы и комплексы и управление ими: материаль VIII междунар. науч.-практ. конф. Новочеркасск, 2008, 4-8. [Levin V.M. Modeling the failure flow of power transformers under operating conditions. Modern energy systems and complexes and their management proceedings of the VIII international science.-prakt. conf. Novocherkassk, 2008. 4-8 (in Russian)]. 